\title{
Acute-onset Autoimmune Hepatitis Treated with Living Donor-liver Transplantation
}

\author{
Keiko Kawai, Kojiro MichitaKA*, Shozo Miyauchi, Masahiro Sano, Masanori Abe, \\ Tsuneyuki NinOMIYA, Bunzo MATSUURA, Toshikazu MASUMOTO, \\ SK MD Fazle AKBAR, Norio HORIIKE and Morikazu ONJI
}

\begin{abstract}
A 50-year-old woman was diagnosed with acute-onset autoimmune hepatitis. She did not respond to steroid therapy including pulse therapy, and was subsequently treated with living donor-liver transplantation 36 days after the beginning of steroid therapy. Except for a period of transient mild acute rejection, her liver function tests remained within a normal range for 2.5 years after the operation. The courses of autoimmune hepatitis patients treated with living-donor liver transplantation have not been previously documented to our knowledge. Living donor-liver transplantation is thought to be one of the therapy options for severe autoimmune hepatitis. (Internal Medicine 42: 158-162, 2003)
\end{abstract}

Key words: rejection, recurrence, severe

\section{Introduction}

Autoimmune hepatitis (AIH) is characterized by the positivity of autoantigen, active hepatitis, a predominance in females, and good response to steroid therapy (1). Liver biopsy specimens show chronic hepatitis or liver cirrhosis with moderate to severe activity in most cases, but some patients have acute hepatitis (2-5). Additionally, it is reported that some acute-onset AIH patients have fulminant or subacute hepatic failure (2-4). Here, we report a patient with acuteonset AIH who progressed to liver failure and was treated with a living donor-liver transplantation (LDLT).

\section{Case Report}

A 50-year-old Japanese woman was admitted to our hos- pital on January 22, 1999 because of jaundice and hepatocellular dysfunction (Fig. 1). In 1994, she had hypergammaglobulinemia, but her liver function test was normal. On January 10, 1999, she felt general fatigue, and on January 17, jaundice had appeared, so she visited a local hospital. Because of a severely abnormal liver function test, she was referred to our hospital. She had no medical history except for a colon polyp that was resected by polypectomy, and she had no history of blood transfusions or drug abuse. She was not a habitual alcohol drinker. No liver disease was found in her family. On admission, physical findings showed jaundice and mild pretibial edema. Ascites was not noted and consciousness was clear. The liver was palpable with 2-finger breadth on the right midclaviclar line. Laboratory data on admission (Table 1) showed total bilirubin $11.1 \mathrm{mg} / \mathrm{dl}$, aspartate aminotransferase (AST) 1,630 IU/l, alanine aminotransferase (ALT) $1,115 \mathrm{IU} / l$, prothrombin time (PT) $35 \%$ of the normal value, albumin $2.8 \mathrm{~g} / \mathrm{dl}$, IgG $3,600 \mathrm{mg} / \mathrm{dl}$, antinuclear antibody (ANA) (1:160, diffuse pattern), and other autoantibodies were negative. Various viral markers, including IgM anti-hepatitis A antibody, hepatitis B surface antigen, IgM anti-hepatitis B core antibody, and anti-hepatitis C antibody, were negative. Hepatitis B virus DNA, hepatitis C virus RNA and hepatitis G virus RNA were negative as determined by polymerase chain reaction. Human leukocyte antigen (HLA) DR typing was 4 and 5. Serum human hepatocyte growth factor level was $1.06 \mathrm{ng} / \mathrm{ml}$. Spaceoccupying lesions, bile duct dilatation, and ascites were not found by ultrasonography. Because of bleeding tendency, a liver biopsy was not performed. The patient's AIH score according to the international criteria (6) was 16 , which was considered to definitely be $\mathrm{AIH}$, so she was diagnosed with AIH.

Initially, steroid pulse therapy (methylprednisolone 1,000 $\mathrm{mg} /$ day for 3 days) was employed followed by oral administration of prednisolone (PSL) at a dose of $60 \mathrm{mg} / \mathrm{day}$.

From the Third Department of Internal Medicine and *the Endoscopy Center, Ehime University School of Medicine, Onsen-gun Received for publication June 19, 2002; Accepted for publication November 22, 2002

Reprint requests should be addressed to Dr. Morikazu Onji, the Third Department of Internal Medicine, Ehime University School of Medicine, Shitsukawa, Shigenobu-cho, Onsen-gun, Ehime 791-0295 


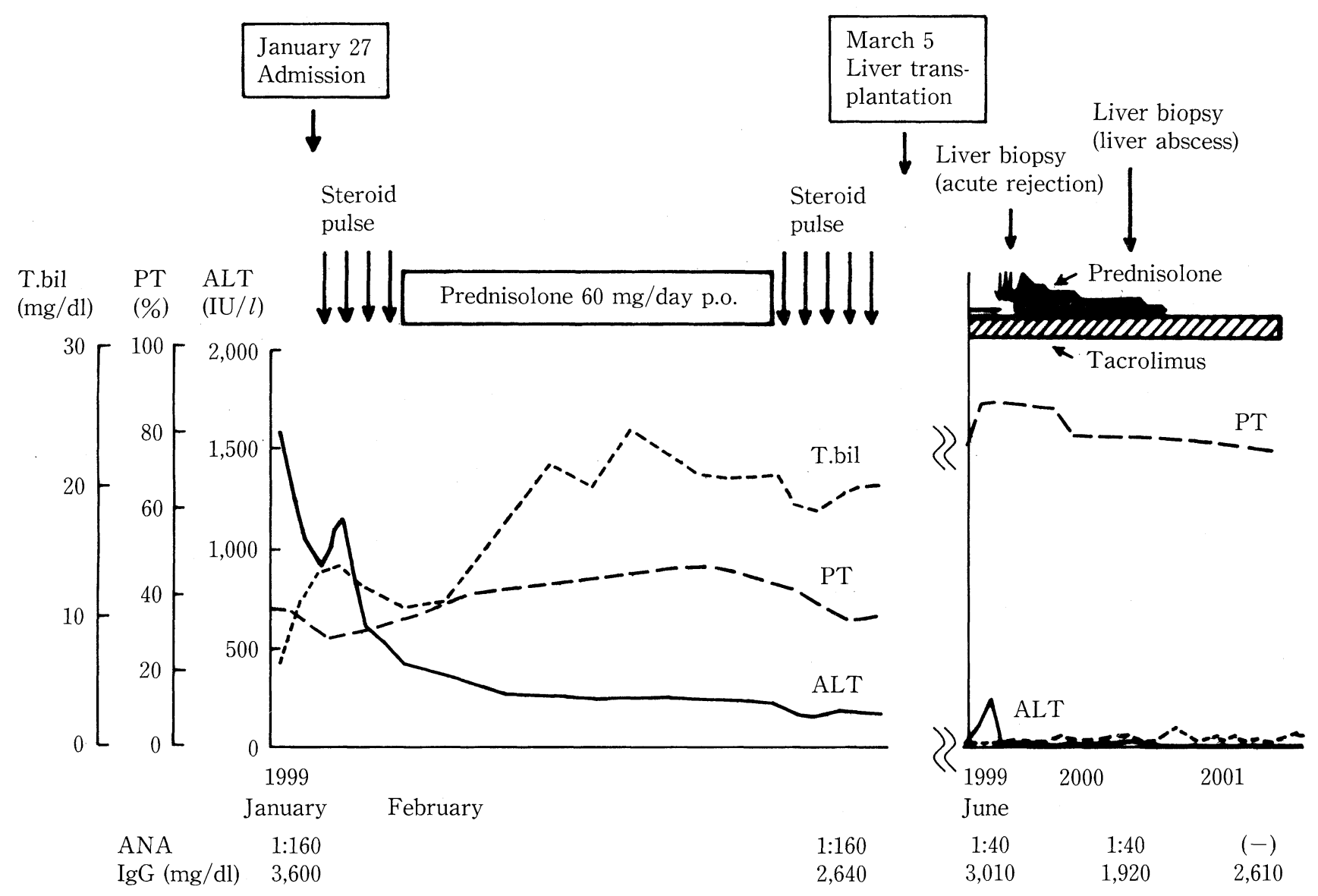

Figure 1. Clinical course in our hospital (1999. January 17 February 26 before liver transplantation, 1999. June $14 \sim 2001$. November after transplantation). The vertical arrow indicates steroid pulse therapy. ALT: alanine aminotransferase, ANA: anti-nuclear antibody, T. bil: total bilirubin, PT: prothrombin time.

Because her consciousness level was clear, we did not perform plasmapheresis or hemodialysis filtration. After the beginning of steroid therapy, transaminases rapidly decreased to $\sim 200-300 \mathrm{IU} / \mathrm{l}$, but total bilirubin increased to $24.4 \mathrm{mg} / \mathrm{dl}$ and PT did not improve. Two weeks after the beginning of immunosuppressive therapy, the disseminated intravascular coagulation (DIC) marker worsened and anorexia emerged. Liver atrophy and ascites were observed, and the low-density area in her liver was enlarged compared to its size on admission as detected by computed tomography. We then performed steroid pulse therapy again. At that time, she did not have infectious symptoms, but fungus was detected by a culture test from her feces and a latex agglutination test from serum. Therefore, we did not add other immunosuppression drugs such as Azathioprine. After steroid pulse therapy, transaminase levels did not improve and bilirubin remained elevated. Her general state became worse and gastrointestinal bleeding emerged. We assessed her condition as indicative of liver transplantation. Informed consent was obtained for LDLT.

The LDLT operation was performed on March 5, 1999 at the Kyoto University Graduate School. Her liver was $925 \mathrm{~g}$, and the transplanted liver from her elder sister's right lobe was $545 \mathrm{~g}$. The body weight and height of the recipient and donor were $62 \mathrm{~kg}, 165.5 \mathrm{~cm}$ and $47 \mathrm{~kg}, 155 \mathrm{~cm}$, respectively. Their blood type was identical. The donor had a good clinical course after the operation.

The liver of the recipient had an uneven, atrophic surface. Histologically, it showed broad collapse of the reticulin framework and bridging necrosis with infiltration of many lymphocytes and plasma cells as well as cholestasis in the lobule (Fig. 2A, B). Most hepatocytes looked severely degenerative, and there was no obvious hepatocyte regeneration. Although there were lymphocytes and plasma cells in the lobule and in the portal area, there were no signs of chronic hepatitis such as obvious scars or fibrosis. Collapses were observed primarily around the central vein areas. The major histological features were compatible with acute-onset AIH. From these histological changes and the clinical course, we diagnosed this case as acute-onset AIH. Although the size of the removed liver was not small, the viable hepatocyte volume was suspected to be small, because the 
KAWAI et al

Table 1. Laboratory Data on Admission

\begin{tabular}{lrlrlr}
\hline WBC & $4,200 / \mathrm{mm}^{3}$ & AST & $1,630 \mathrm{IU} / l$ & ANA & $\times 160$ (DI) \\
RBC & $3,600,000 / \mathrm{mm}^{3}$ & ALT & $1,115 \mathrm{IU} / l$ & LE test & $(-)$ \\
Hemoglobin & $11.4 \mathrm{~g} / \mathrm{dl}$ & LDH & $728 \mathrm{IU} / l$ & AMA & $<20$ \\
Hematoclit & $34.9 \%$ & ALP & $268 \mathrm{IU} / l$ & anti-LKM-1 & $(-)$ \\
Platelet & $55,000 / \mathrm{mm}^{3}$ & $\gamma$-GPT & $92 \mathrm{IU} / l$ & AFP & $87 \mathrm{ng} / \mathrm{ml}$ \\
Prothrombin time & $35 \%$ & ChE & $79 \mathrm{IU} / l$ & HGF & $1.06 \mathrm{ng} / \mathrm{ml}$ \\
Anti-thrombin III & $7.1 \mathrm{mg} / \mathrm{dl}$ & LAP & $118 \mathrm{IU} / l$ & IgM anti-HA & $(-)$ \\
Fibrinogen & $112 \mathrm{mg} / \mathrm{dl}$ & ZTT & $19 \mathrm{U}$ & HBsAg & $(-)$ \\
FDP D-dimer & $2.7 \mu \mathrm{g} / \mathrm{dl}$ & TTT & $20 \mathrm{U}$ & IgM anti-HBc & $(-)$ \\
ESR $(1 \mathrm{~h})$ & $17 \mathrm{~mm}$ & BUN & $4 \mathrm{mg} / \mathrm{dl}$ & HBV-DNA & $(-)$ \\
Total protein & $7.4 \mathrm{~g} / \mathrm{dl}$ & Cr & $0.7 \mathrm{mg} / \mathrm{dl}$ & anti-HCV & $(-)$ \\
Albumin & $2.8 \mathrm{~g} / \mathrm{dl}$ & FPG & $126 \mathrm{mg} / \mathrm{dl}$ & HCV-RNA & $(-)$ \\
Total bilirubin & $11.1 \mathrm{mg} / \mathrm{dl}$ & NH & $107 \mu \mathrm{gg} / \mathrm{dl}$ & HGV/GBV-C RNA & $(-)$ \\
Direct bilirubin & $7.1 \mathrm{mg} / \mathrm{dl}$ & IgG & $3,600 \mathrm{mg} / \mathrm{dl}$ & HLA DR DR4, DR5 & \\
& & IgA & $286 \mathrm{mg} / \mathrm{dl}$ & & \\
& & IgM & $137 \mathrm{mg} / \mathrm{dl}$ & & \\
\hline
\end{tabular}

AFP: alpha-fetoprotein, ALT: alanine aminotransferase, AMA: anti-mitochondrial antibody, ANA: anti-nuclear antibody, anti-HA: anti-hepatitis A, AST: aspartate aminotransferase, FDP: fibrin/ fibrinogen degradation products, ESR: erythrocyte sedimentation rate, HBc: hepatitis B core, HBsAg: hepatitis B virus surface antigen, HBV: hepatitis B virus, HCV: hepatitis C virus, HGF: hepatocyte growth factor, HGV: hepatitis G virus, HLA: human leukocyte antigen, LKM: liver-kidney microsome.

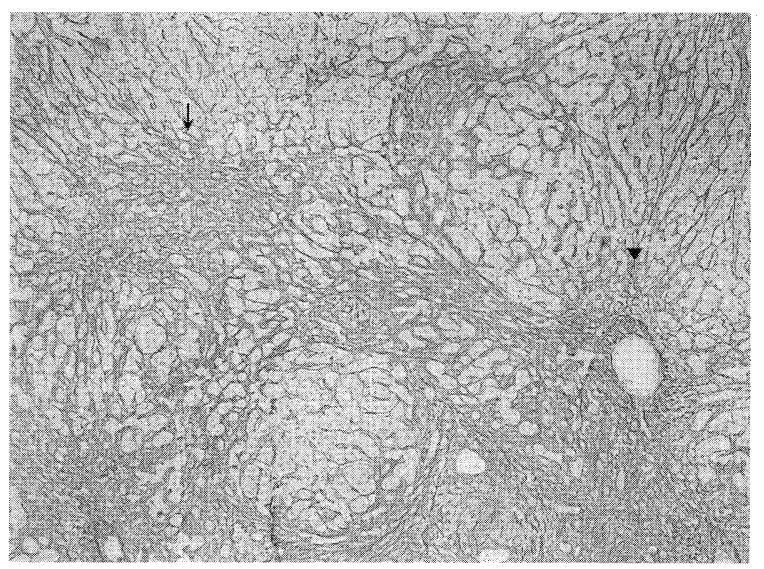

A

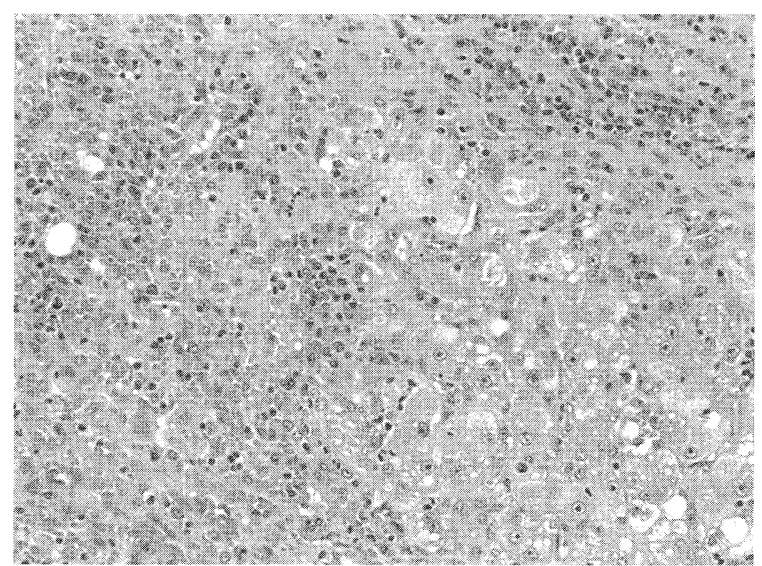

B

Figure 2. A. Histological findings of the removed liver revealed broad collapse of the reticulin framework and bridging necrosis. Central vein area and portal area are indicated by arrowhead and arrow, respectively. Collapses were observed primarily around the central vein areas. There were no signs of chronic hepatitis such as obvious scars or fibrosis (silver stain, $\times 40$ ). B. Infiltration of many lymphocytes and plasma cells is shown in the lobule and the portal area, and cholestasis was present. Most hepatocytes looked severely degenerated (HE stain, $\times 400)$.

hepatocyte area was small in histological specimens observed under the light microscope.

After the liver transplantation, liver function tests were normal, and except for a period of transient mild acute rejection, liver function tests remained within the normal range for 2.5 years after the operation. Additionally, there were no problems in the donor's clinical course following the operation. The ANA was positive (1:160) before the operation, and its level decreased (1:40) 3 months after the operation and became negative 16 months after the operation. Prior to the operation, the IgG level, which was $3,600 \mathrm{mg} / \mathrm{dl}$ on admission, only decreased to $2,640 \mathrm{mg} / \mathrm{dl}$ after steroid therapy, which is still relatively high. This level gradually decreased to normal 6 months after the operation. The 
patient had a liver abscess 1 year after the operation, but it was resolved by the administration of antibiotics and by discontinuation of prednisolone. The IgG level has now increased to $2,720 \mathrm{mg} / \mathrm{dl}$. Liver biopsy was performed at 4 months and at 1 year after the operation. The former specimen showed evidence of mild acute rejection. However, no evidence of AIH recurrence was observed in either specimen.

\section{Discussion}

AIH is generally effectively treated with immunosuppressive therapy including steroid therapy; however, some AIH cases that present as fulminant hepatitis or as a severe form of acute hepatitis are reported to have a poor prognosis, such as that of the subacute type of fulminant hepatitis that is caused by hepatitis virus $(2-5,7)$. It is difficult to diagnose these cases based on the international criteria, because these criteria are based on the clinical data of AIH patients with chronic hepatitis and liver cirrhosis $(2,6)$.

It is reported that factors associated with rapid progression of AIH include no concurrent autoimmune disease, a DR4negative phenotype, being female, and positivity of the antiliver-kidney-microsome 1 (anti-LKM 1) antibody (8). In this case, there is no concurrent autoimmune disease but the patient is DR4-positive, female, and anti-LKM 1 antibodynegative. This patient did not appear to have hepatic encephalopathy. Although she received steroid therapy including steroid pulse therapy for 1 month, PT and bilirubin levels did not improve, DIC continued, and complications including gastrointestinal bleeding and ascites emerged. Failure of the serum bilirubin level to improve after 2 weeks of corticosteroid therapy in a patient with multilobular necrosis is variably associated with death (9). This patient fulfilled the King's College criteria for liver transplantation (10). Additionally, the high-dose steroid therapy would be expected to increase the risk of infection for a long time, making the need for liver transplantation more urgent. The right lobe of a donor was used as a graft. Auxiliary partial orthotopic living donor liver transplantation (APOLT) was considered, but the graft size in this case was sufficient.

AIH patients that receive a cadaveric liver transplantation have an excellent survival rate. Histologically AIH recurrence occurred in $20-42 \%$ of patients (11-17). Most reported AIH recurrence episodes were mild, although some severe cases that required retransplantation have been documented (14). Autoantibodies persist to some extent following liver transplantation in most cases (11-13). The correlation between antibody persistence and graft dysfunction is unclear. Reich et al reported that $\mathrm{AIH}$ recurrence occurred less often in patients who presented with fulminant hepatic failure compared to those who had known chronic disease (14). Acute rejection after liver transplantation occurs in up to $80 \%$ of AIH patients $(11,14-16)$ and is sometimes resistant to steroid treatment.

In Japan, cadaveric liver transplantation is performed less often than LDLT due to the shortage of donor grafts. The donor for this patient was an elder sister with identical blood type. The incidence of AIH recurrence is not known in patients with autoimmune hepatitis who received LDLT from a relative. At the present time, this patient has not had an AIH recurrence for 2.5 years after the operation, although she had a transient mild acute rejection. To our knowledge, this is the first report about the course of an acute-onset AIH patient who was treated with a living donor-liver transplantation. Many more cases need to be observed to support the observations in this study.

Acknowledgements: The authors gratefully acknowledge Prof. Tanaka Koichi and the surgeons in the Department of Transplantation Surgery, Kyoto University Graduate School of Medicine.

\section{References}

1) Czaja AJ, Davis GL, Ludwig J, Baggenstoss AH, Taswell HF. Autoimmune features as determinants of prognosis in steroid-treated chronic active hepatitis of uncertain etiology. Gastroenterology $\mathbf{8 5}$ : 713-717, 1983.

2) Abe M, Hiasa $Y$, Masumoto $T$, et al. Clinical characteristics of autoimmune hepatitis with histological features of acute hepatitis. Hepatol Res 21: 213-219, 2001.

3) Burgart LJ, Batts KP, Ludwig J, Nikias GA, Czaja AJ. Recent-onset autoimmune hepatitis. Biopsy findings and clinical correlations. [Published erratum appears in Am J Surg Pathol 19: 1341, 1995] Am J Surg Pathol 19: 699-708, 1995.

4) Redondo-Cerezo E, Nogueras-Lopez F, Martin-Vivaldi R, Simon EE. Fulminant hepatic failure as the presenting form of type 1 autoimmune hepatitis in the elderly. Am J Gastroenterol 97: 1265-1266, 2002.

5) Herzog D, Rasquin-Weber AM, Debray D, Alvarez F. Subfulminant hepatic failure in autoimmune hepatitis type 1: an unusual form of presentation. J Hepatol 27: 578-582, 1997.

6) Alvarez F, Berg PA, Bianchi FB, et al. International Autoimmune Hepatitis Group Report: review of criteria for diagnosis of autoimmune hepatitis. J Hepatol 31: 929-938, 1999.

7) Nakadate I, Nakamura A, Endo R, et al. Autoimmune hepatitis presenting with acute hepatic failure. Kanzo (Acta Hepat Jpn) 34: 665-671, 1993 (in Japanese, Abstract in English).

8) Milkiewicz P, Ahmed M, Hathaway M, Elias E. Factors associated with progression of the disease before transplantation in patients with autoimmune hepatitis. Liver 19: 50-54, 1999.

9) Czaja AJ, Rakela J, Ludwig J. Features reflective of early prognosis in corticosteroid-treated severe autoimmune chronic active hepatitis. Gastroenterology 95: 448-453, 1988.

10) O'Grady JG, Alexander GJ, Hayllar KM, Williams R. Early indicators of prognosis in fulminant hepatic failure. [see comments] Gastroenterology 97: 439-445, 1989.

11) Ratziu V, Samuel D, Sebagh M, et al. Long-term follow-up after liver transplantation for autoimmune hepatitis: evidence of recurrence of primary disease. J Hepatol 30: 131-141, 1999.

12) Prados E, Cuervas-Mons V, de la Mata M, et al. Outcome of autoimmune hepatitis after liver transplantation. Transplantation 66 : 1645-1650, 1998.

13) Milkiewicz P, Hubscher SG, Skiba G, Hathaway M, Elias E. Recurrence of autoimmune hepatitis after liver transplantation. Transplantation 68: 253-256, 1999.

14) Reich DJ, Fiel I, Guarrera JV, et al. Liver transplantation for autoimmune hepatitis. Hepatology 32: 693-700, 2000.

15) Götz G, Neuhaus R, Bechstein WO, et al. Recurrence of autoimmune hepatitis after liver transplantation. Transplant Proc 31: 430431, 1999. 


\section{KAWAI et al}

16) Narumi S, Hakamada K, Sasaki M, et al. Liver transplantation for autoimmune hepatitis: rejection and recurrence. Transplant Proc 31: 1955-1956, 1999.
17) Ayata G, Gordon FD, Lewis WD, et al. Liver transplantation for autoimmune hepatitis: a long-term pathologic study. Hepatology 32: 185-192, 2000. 